\title{
Acute idiopathic pericarditis and calcific aortic stenosis: unusual fatal disease combination
}

\author{
Charles E. Wheatley, M.D.* \\ Ann Arbor, Mich.
}

Acute idiopathic pericarditis with effusion is rarely fatal unless it complicates chronic myocardial disease. Acute epimyocarditis and the impedance to ventricular filling due to pericardial tamponade further decreases the low cardiac output due to primary myocardial, valvular, hypertensive, or atherosclerotic heart disease. Treatment of acute pericarditis with effusion in the presence of chronic myocardial disease may be very difficult. This presentation of a fatal case illustrates these truths.

\section{Case report}

D. N., a 42-year-old white male Michigan National Guardsman, worked 36 consecutive hours on May 11 and 12, 1973, despite shorness of breath, to help his company prepare for weekend maneuvers. He was fatigued and febrile the following day. Dyspnea and cough productive of white, frothy sputum developed after he walked 30 feet. His oral temperature rose to $103^{\circ} \mathrm{F}$. during the next 5 days. Dyspnea with cough at rest and a constant, aching anterior chest pain, which was aggravated by deep inspiration, chest wall movement, and the supine position, appeared. Tetracycline, penicillin, and hydrochlorothiazide were prescribed because of basilar râles and "precordial blowing systolic and apical diastolic murmurs." An electrocardiogram showed left ventricular enlargement. He was admitted to hospital on May 23, 1973.

An illness from which he recalled no symploms forced his absence from school for 1 year at age 12. Inactive rheumatic heart disease with mitral stenosis was diagnosed at age 32 after a "systolic murmur with a diastolic rumble," was heard and cardiac fluoroscopy showed cardiac calcification. An electrocardiogran was normal at that time, but tracings in 2971 showed left ventricular enlargement. In 1972 snow

From the University of Michigan Niedical Center, Ann Arbor, Mich. Received for publication May 20, 1977.

Accepted for publication July 28, 1977.

Reprint requests: C. E. Wheatley, M.D., Division of Cardiology (Heart Station), University of Michigan Medical Center, Ann Arbor, Mirh. 48109

*Clinical Instructor in Internal Medicine, University of Michigan Medical Center. shoveling caused chest "fullness" which disappened with rest. Caugh, palpitations, mild shortness of breath, and fever appeared in February, 1973, but remitted after 3 days of bedrest. Dizziness or syncope never occurred, although he intermittently performed strenuous work.

Admission physical examination showed a chronically ill, pale, mildly diaphoretic and moderately dyspnetc white male with temporal muscle wasting who was unable to recline below 60 degrees due to aggravation of his chest pain and dyspnea. The blood pressure was $105 / 5045$ both arme sitting, temperature $99.8^{\circ} \mathrm{F}$. (oral), heart rate $90 / \mathrm{minuta}$, respiratory rate 22 /minute. Neck venous distention at 60 degrees was minimal: venous waves, paradoxical pulse, anj Kussmaul's sign were absent in all positions. Bilateval harsh carotid and subclavian transriatted systolic murmurs were present. Carotid upstrokes were delayed but equal. Eye, ear, nose, and throat examinations were normal. There were fine wet râles over the left lateral chest and bilateral basilar decreased vocal fremitus.

The left border of cardiac dullness was $15 \mathrm{~cm}$. beyond the midsternal line, $2 \mathrm{~cm}$. beyond the midclavicular line, and the point of maximum impulse was diffuse in the sixth intercostal space. The heart sounds could not be heard at the base and were greatly reduced along the left sternal borrder and at the apex. Exba sounds were not heard. A regular whythm at 68 beats per minute was present with occasional premature beats. A harsh crescendo-decrescendo systolic murmur at the base became more high-pitched along the lower left stemal border and radiated to the apex, infraclavicular, and interscapular areas. A high-pitched early and mid-diastolic desrescendo murmur was present along the ieft sternal border. A pericardial knock and continuous friction rub were heard at the third and fourth intercostal spaces, left sternal border, and at the apex. A slightly tender liver with a total height of $1 \frac{4}{4} \mathrm{~cm}$. was palpable $3 \mathrm{~cm}$, below the right costal margin, but the spleen and kidneys were not palpable. Abdominal masses. bruits, and tenderness were absent. The dorealis pedis pulses were diminished, but the other peripheral pulses were bounding and equal. Peripheral edema, cyarosis, clubbing, and skin or nail changes to suggest bacterial endocarditis were absent.

Elevation of leukocyte counts from 11,000 io $23,000 / \mathrm{mm}^{3}$ with left shift and toxic granulation suggester infection but repeat cultures of urine, stool, and blood for bacteria (including $M$. thberculosis), fungi, yeasts, and viral agencs were 


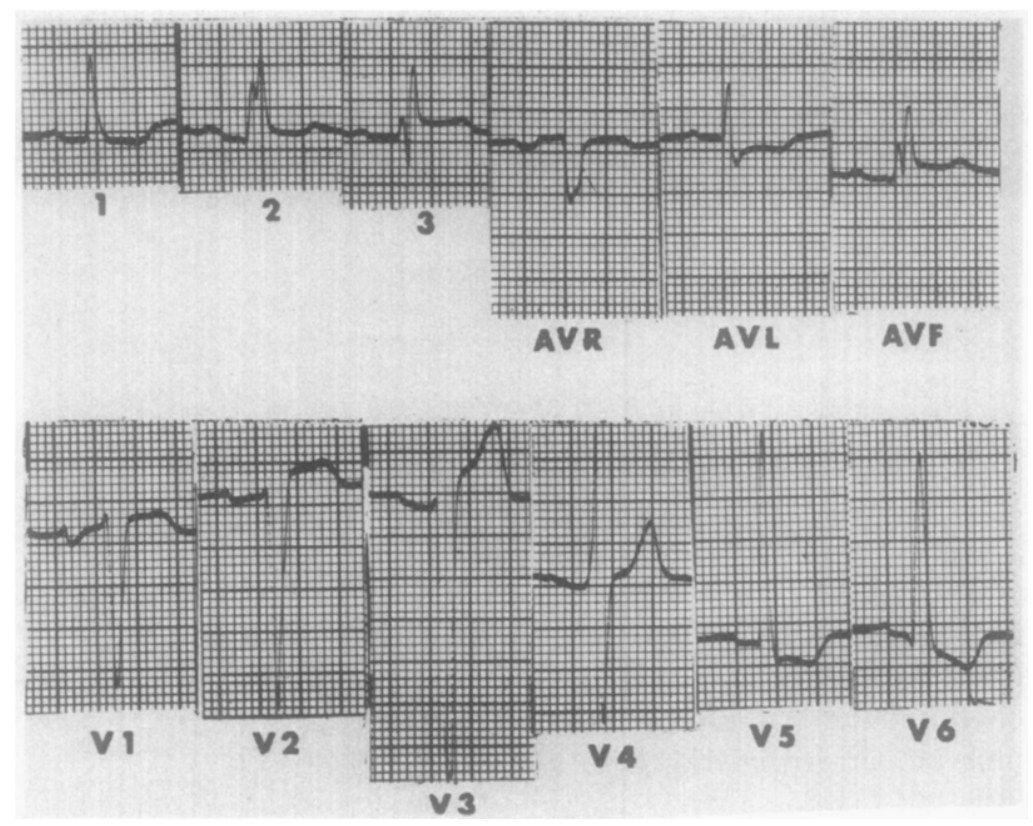

Fig. 1. ECG before pericardiectomy.

negative. Sputum cultures grew Candida albicans and Klebsiella species. Hemoglobin $11 \mathrm{gm}$. per cent, plasma, hemoglobin $3.6 \mathrm{mg}$. per cent, serum iron $37 \mu \mathrm{g}$ per cent, total iron binding capacity $330 \mu \mathrm{g}$ per cent, serum haptoglobin less than 20 units suggested hemolysis and iron deficiency. Screening tests for thyroid and renal disease, lupus erythematosus, and rheumatoid arthritis were negative. Immunoglobulin electrophoresis showed elevated IgA. Abnormal enzyme values were serum amylase 135 units, glutamic oxalacetic transaminase 147 to 202 units, glutamic pyruvic transaminase 246 units, lactic dehydrogenase 318 units with increased fast fractions 1 , 2. Serum total bilirubin was $2.8 \mathrm{mg}$. per cent. Electrocardiograms showed left atrial depolarization abnormality, first degree atrioventricular block, left ventricular enlargement, and ST elevation suggestive of pericarditis (Fig. 1). Admission chest $\mathrm{x}$-ray showed massive, water-bottle enlargement of the cardiac silhouette, typical of pericardial effusion and mild venous congestive changes (Fig. 2).

Right heart catheterization findings (Table I) showed greater elevation of pulmonary artery diastolic pressure than severely elevated right atrial and ventricular diastolic pressures, which suggested that left ventricular failure was a major cause of the low cardiac output. After $\mathrm{CO}_{2}$ and dye contrast, right atrial angiocardiograms demonstrated a large pericardial effusion (Fig. 3); pericardiocentesis yielded a serosanguineous fluid. The patient remained very weak, anoretic, and tachypneic despite this procedure, and an $8 \mathrm{lb}$. diuresis induced by digitalis and diuretics. Pericardiectomy with removal of a large sanguineous effusion was performed.

Pericardial fluid and tissue cultures were negative for all pathogens. The oral temperature was $100.5^{\circ} \mathrm{F}$. at 4 P.M. for three consecutive days after surgery and then remained normal. Dyspnea and orthopnea improved shortly after surgery, but severe dyspneic episodes were precipitated later by activity such as use of bed pan. Despite a $25 \mathrm{lb}$. weight loss, pulmonary congestion increased and bilateral fluffy inflltrates were seen on chest $x$-ray. Pulmonary embolism could not be differentiated from edema by perfusion lung scan.

Due to intractable congestive heart failure, the patient was transferred to the University of Michigan Hospital on July 2, 1973 , for possible aortic valve replacement. Admission physical examination confirmed previous findings and revealed an erythematous area over the hard palate and several vesicular lesions on the left buccal mucosa. Urinary output and orthopnea improved after hyperalimentation, which was discontinued after central venous pressure reached $22 \mathrm{~cm} . \mathrm{H}_{2} \mathrm{O}$. He was treated for suspected septicemia with penicillin-G 10 million units daily and streptomycin $1.0 \mathrm{Gm}$. daily. Thirteen days after admission the diastolic blood pressure dropped to $30 \mathrm{~mm}$. $\mathrm{Hg}$ and short episodes of substernal pressure-like chest pain occurred.

Left heart catheterization (Table I) showed mild mitral insufficiency, $65 \mathrm{~mm}$. $\mathrm{Hg}$ gradient across a heavily calcified aortic valve, and a hypertrophic, moderately hypokinetic left ventricle. The severely calcified stenotic aortic valve was replaced with a Starr-Edwards ball prosthesis, but primary closure of the aorta was unsuccessful and a Dacron prosthetic graft was used to replace the ascending aorta. Pulmonary edema developed during parallel cardiopulmonary bypass. Return to full bypass and furosemide did not produce diuresis; ventricular fibrillation developed, and defibrillation was unsuccessful.

\section{Tissue findings.}

A. Surgical The pericardium was $.4 \mathrm{~cm}$. thick. Soft, brown, papillary excrescences were seen. Microscopic sections showed marked fibrosis and scattered inflammatory infiltrate composed mostly of lymphocytes with tibrin attached to one surface.

B. Autopsy. (Limited to thorax with transdiaphragmatic liver biopsy). Chronic calcific aortic valvulitis. Normal mitral valve. Fibrinous epicarditis and myocarditis (Fig. 4). Biventricular myocardial hypertrophy (Fig. 5) with focal fibrosis 


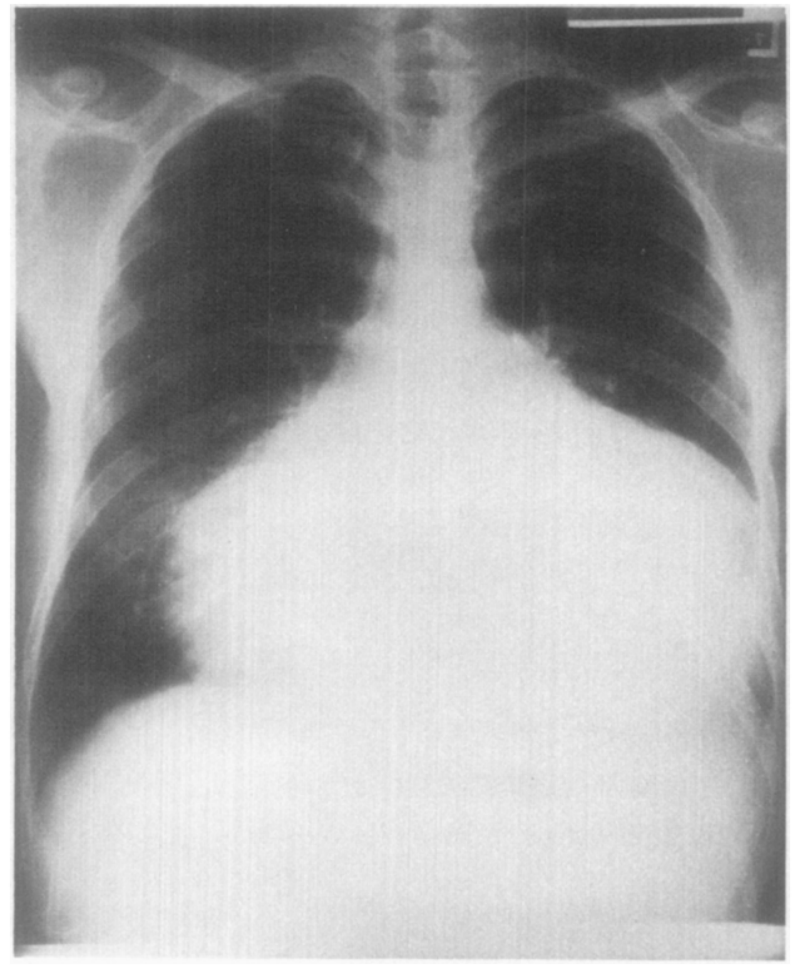

Fig. 2. Admission chest x-ray, before pericardiectomy.

and necrosis of inyocardium and absence of inflammatory interstitial infiltrate. Pulmonary edema and pulmonary arterial embolus. Chronic passive congestion of liver with centrilobular necrosis and fatty inflltration. Granulomatous mediastinal lymphadenopathy.

\section{Discussinon}

Acute idiopathic pericarditis is usually benign, although frequently recurrent. This is not true if other chronic myocardial disease is present. Liu and Garcia's review ${ }^{1}$ of the literature to 1965 reported 12 fatal cases of acute idiopathic pericarditis. Cardiac tamponade was thought to be the cause of death in five but severe chronic myocardial disease was present in six others. Aortic stenosis with myocardial hypertrophy was present in one of these six cases. An additional fatal case of idiopathic pericarditis complicating calcareous aortic stenosis and cardiac hypertrophy was reported by Kirschner and associates in 1971. Their patient also had acute subendocardial (zonal) infarction without extension to the outer one half of the myocardium. Scarch of the English literature since 1965 did not reveal additional cases of this fatal combination of diseases.

Before the advent of $\mathrm{CO}_{2}$ and dye contrast

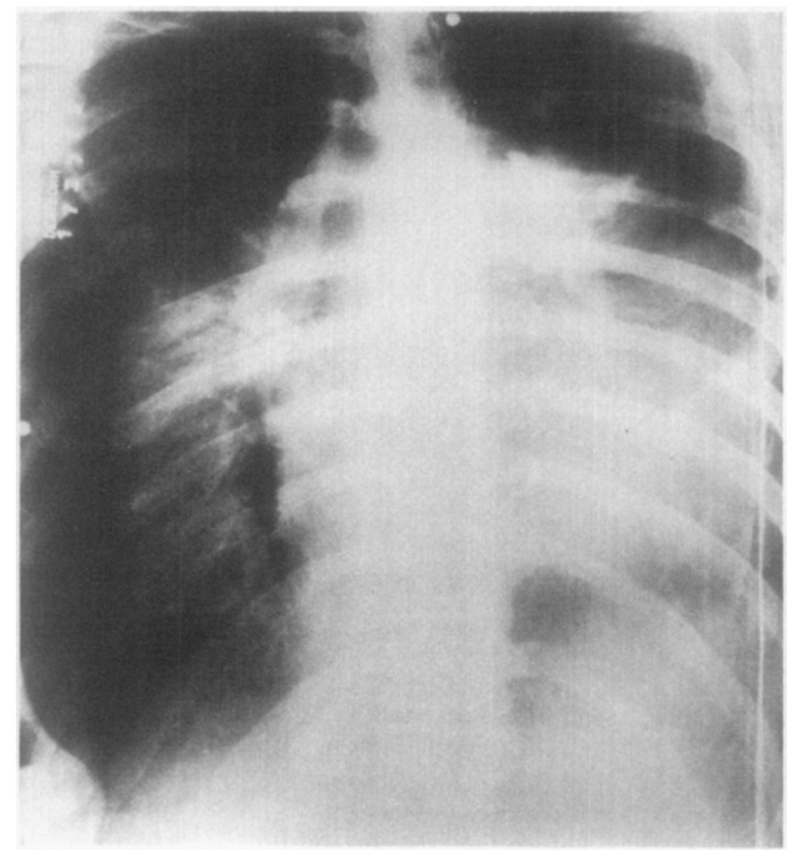

Fig. 3. $60_{2}$ righ atral angiocardiogram. Sarge pericardio effusion separates $\mathrm{CO}_{2}$ in right atrium and right heart border.

Table 1. Catheterization findings

\begin{tabular}{|c|c|c|}
\hline Site & $\begin{array}{c}\text { Before } \\
\text { pericardiectomy } \\
\text { pressure (mm. Hg) }\end{array}$ & 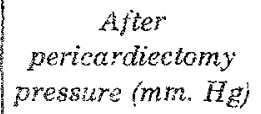 \\
\hline 1. Right atriurn & $40 / 30$ Mean $=35$ & $\mathrm{Agen}=18$ \\
\hline 2. Right ventricle & $100 / 20$ RVED $=30$ & $85 / 0 \mathrm{RVED}=20$ \\
\hline $\begin{array}{l}\text { 3. Main pulmonary } \\
\text { artery }\end{array}$ & $110 / 65$ Mean $=70$ & $100 / 30$ Mean $=45$ \\
\hline $\begin{array}{c}\text { 4. Left pulmonary } \\
\text { artery wedge }\end{array}$ & - & 25 \\
\hline 5. Brachial artery & - & $\$ 50 / 40$ \\
\hline 6. Ascending aorta & - & $85 / 40$ \\
\hline 7. Left ventricle & - & $150 / \mathrm{LVED}=28$ \\
\hline
\end{tabular}

angiography and echocardiography, the diagnosis of pericardial effusion in the presence of other cause for the enlargement of the heart was ofter made at autopsy. ${ }^{3}$ When two diseases that affect myocardial function are known to be present, determination of the degree that each disease contributes to the low cardiae output may be extremely difficult. * The contribution that acute opimyocarditis makes to myocardial dysfunction is speculative, although myocardial biopsy may have demonstrated myocarditis. Fericardial effusion with or without tamponade may mask the changes on physical examination that 


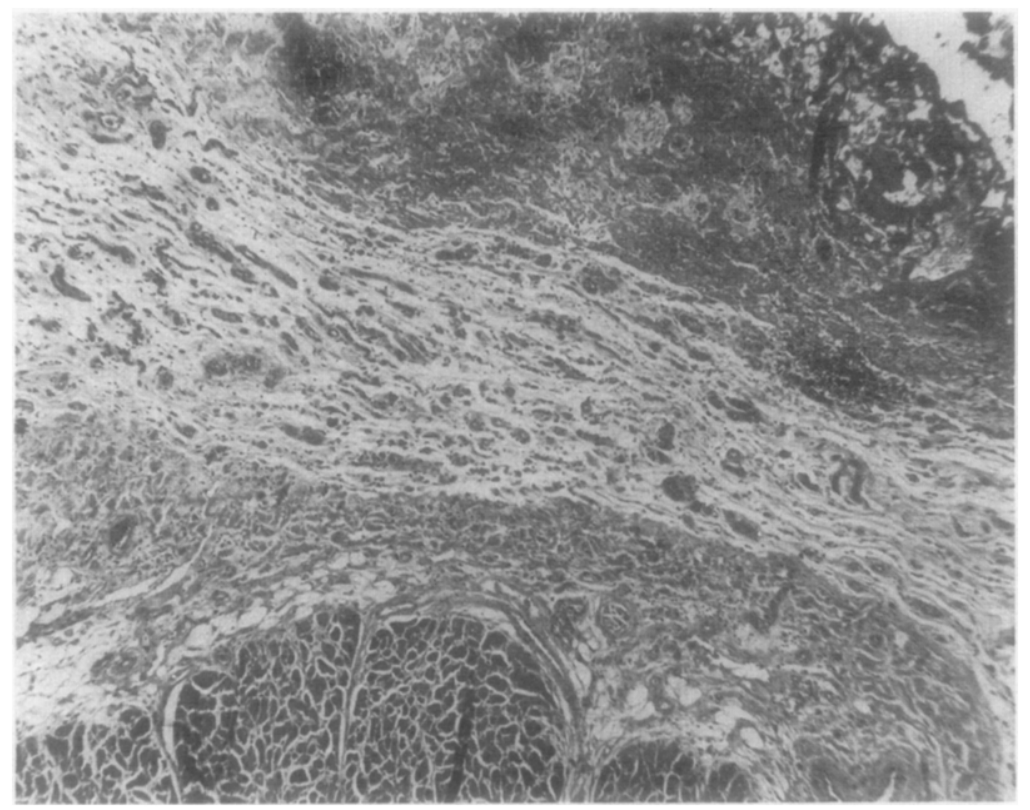

Fig. 4. Visceral pericardium (top right) and epicardium with fibrinous epicarditis and myocarditis.

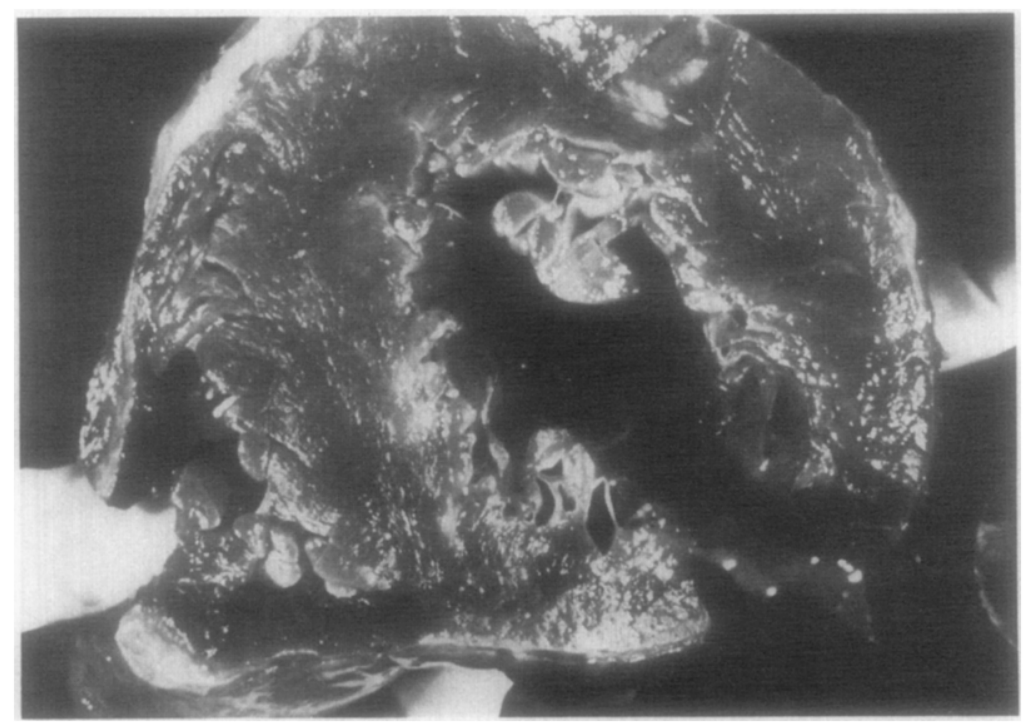

Fig. 5. Gross specimen, left and right ventricular enlargement.

indicate antecedent ventricular failure. Pulsus paradoxus may not be produced by tamponade in the presence of aortic stenosis, due to the failure of transmission of respiratory variations in left ventricular systolic pressure to the arterial system. ${ }^{5,6} \mathrm{X}$-ray findings of clear lung fields and a massively enlarged cardiac silhouette (Fig. 1) suggest tamponade, but changes on physical examination that indicate ventricular enlargement point more accurately to the cause of the greatest hemodynamic abnormality. Pericardiec- tomy removes a lax effusion, but unless tamponade is present, more severe congestive failure may follow. Lange and colleagues ${ }^{6}$ found that abnormal hemodynamic changes in nine patients with lax effusion did not improve after pericardiectomy in contrast to improvement in similar parameters in nine patients with tamponade. Berglund and associates ${ }^{7}$ state that the left ventricle stressed from outflow obstruction, as in aortic stenosis, dilates and produces increased pericardial pressure which limits right ventricular dia- 
stolic expansion and output: the consequences are lower pulmonary capillary and left atrial pressures than would result if the pericardium were not present. Pcricardiectomy, by permitting greater pulmonary blood flow, may be followed by increased left heart failure.

The clinician faces a dilemma when surgery is considered for acute pericarditis with effusion in the presence of left ventricular hypertrophy due to aortic stenosis. Surgery may relieve the dominant cause for the low cardiac output if tamponade is present, but if the chronic myocardial disease is the major cause for the low cardiac output, surgery may tip the balance unfavorably, although valve replacement may correct the primary cause of the chronic myocardial disease.

\section{Summary}

Acute idiopathic pericarditis with massive pericardial effusion complicated left ventricular enlargement due to calcific aortic stenosis and led to intractable congestive heart fallure and death during aortic valve replacement in a 42 year-old white male. This unusual combination of acute and chronic diseases is lethal due to their combined deleterious effect upon myocardial function.

\section{REFERENCES}

1. Liu, H. Y. and Garcia, R.: Acute idiopathe pericarditis, AM. HearT J. 69:677, 1965.

2. Kirschner, R. H., Archer, F. L., Page, M., at al., Clinical pathologic conference, AM. HEART I. $81.564,1971$.

3. Case Records of the Massachusetts General Hospital, N. Engl. J. Med. 258:1004, 1958.

4. Burch, Gr. E., and Phillips, J. H.: Methods in the diagnosic differentiation of myocardial dilatation from perichrdial effusion, AM HEART I 64:266, 1962.

5. Lange, R. L.: Compressive cardiac and circulatory disorders: Clinical and laboratory correlation, AM. HART J. 74:419, 1967.

6. Lange, R. L., Botticelli, J. T., Tsagaris, T. I., et al. Diagnostic signs in compressive cardiac disorders, Circulation 23:763, 1966.

7. Berglund, E., Sarnoff, S. J., and Isaacs, J. P.: Role of the pericardium in regulation of cardiovascular hemodynamics, Circ. Res. 3:133, 1955. 